\title{
OVERVIEW ON ASSOCIATION OF DIFFERENT TYPES OF LEUKEMIAS WITH RADIATION EXPOSURE
}

\author{
D.F. Gluzman*, L.M. Sklyarenko, M.P. Zavelevich, S.V. Koval, T.S. Ivanivska, N.K. Rodionova \\ R.E. Kavetsky Institute of Experimental Pathology, Oncology and Radiobiology, NAS of Ukraine, \\ Kyiv 03022, Ukraine
}

\begin{abstract}
Exposure to ionizing radiation is associated with increasing risk of various types of hematological malignancies. The results of major studies on association of leukemias and radiation exposure of large populations in Japan and in Ukraine are analyzed. The patterns of different types of leukemia in 295 Chernobyl clean-up workers diagnosed according to the criteria of up-to-date World Health Organization classification within 10-25 years following Chernobyl catastrophe are summarized. In fact, a broad spectrum of radiation-related hematological malignancies has been revealed both in Life Span Study in Japan and in study of Chernobyl clean-up workers in Ukraine. The importance of the precise diagnosis of tumors of hematopoietic and lymphoid tissues according to up-to-date classifications for elucidating the role of radiation as a causative factor of leukemias is emphasized. Such studies are of high importance since according to the recent findings, radiation-associated excess risks of several types of leukemias seem to persist throughout the follow-up period up to 55 years after the radiation exposure.
\end{abstract}

Key Words: ionizing radiation, hematological malignancies, leukemia risk, Chernobyl clean-up workers.

\section{RISK OF LEUKEMIA FOLLOWING RADIATION EXPOSURE: BACKGROUND INFORMATION}

Increasing risk of leukemia and cancer is believed to represent the most serious late effect of the exposure to ionizing radiation. The risks of hematological malignancies related to the acute or protracted low dose or low dose rate of radiation exposure in various populations have been assessed in several studies. In particular, the risks of leukemias and other types of hematological malignancies in A-bomb survivors have been the subject of on-going studies conducted at Radiation Effects Research Foundation (RERF), Research Institute for Nuclear Medicine and Biology Hiroshima University, and Atomic Bomb Disease Institute Nagasaki University School of Medicine [1-3]. In fact, leukemia was the first malignancy linked to radiation exposure in atomic bomb survivors. Much has been learned about leukemia incidence and mortality in RERF Life Span Study (LSS) cohort of A-bomb survivors [1, 3]. The results of several studies on association between leukemia and radiation exposure in Chernobyl clean-up workers and population of contaminated areas have been also presented $[4,5]$.

The relative leukemia risk has been shown to increase directly proportional to the bone marrow dose. For leukemia cases diagnosed in Japan, this relationship was particularly striking for chronic myeloid leukemia $(\mathrm{CML})$ and acute lymphoblastic leukemia (ALL) indica-

\begin{tabular}{|c|c|}
\hline & \\
\hline Corresponde & $\begin{array}{l}\text { E-mail: vals@onconet.kiev.ua; } \\
\text { mzavelevych@yahoo.com }\end{array}$ \\
\hline
\end{tabular}

Abbreviations used: ALL - acute lymphoblastic leukemia; $\mathrm{AML}$ - acute myeloblastic leukemia; CLL - chronic lymphocytic leukemia; CML - chronic myeloid leukemia; ERR - excess relative risk; LGL - large granular lymphocytic leukemia; LSS - Life Span Study; MDS - myelodysplastic syndromes; MM - multiple myeloma; NHL - non-Hodgkin's lymphoma; RERF - Radiation Effects Research Foundation. ting the close association between ionizing radiation and the induction of these types of leukemia. The association between radiation exposure and acute myeloblastic leukemia (AML) has been also proved $[1,6]$.

Excess relative risk (ERR) per unit of radiation for leukemia appears to be one of the highest among all radiation-associated cancers [7]. However, the absolute number of leukemia cases even in such large cohorts as A-bomb survivors and Chernobyl clean-up workers is low because leukemia incidence is much lower than that of the most prevalent solid cancers (leukemia accounts for only $4 \%$ of all cancer deaths in LSS).

The patterns of radiation exposure of human populations including the mode of radiation, the contribution of internal versus external radiation, the cumulative dose and dose rate, etc. varied significantly. Nevertheless, the total relative risk assessments of leukemias in Japanese atomic bomb survivors [3] and in occupationally exposed nuclear industry workers [8] seemed to be within the same order of magnitude. The ERR of 4.9 per 1 Gy was assessed in the general populations living in Ural area contaminated with radionuclides [9]. Moreover, 32-48\% of all leukemia cases in these studies were estimated as attributable to radiation exposure [3, 9]. In Russian cohort of Chernobyl clean-up workers, Ivanov et al. [10] demonstrated the time-averaged ERR per 1 Gy for leukemias slightly exceeding that in LSS cohort in Japan.

The excess radiation-induced leukemias should be evident in about two years after radiation exposure with excess peak at about 7-8 years after exposure. In fact, the systematic analysis of leukemia incidence and mortality in Hiroshima and Nagasaki was launched in 5 years following A-bombing, so a significant number of early cases was probably missed. The same holds true for leukemia study in Chernobyl cleanup workers and other categories of people suffered after Chernobyl since the collection of data and their 
systematical analysis has not started earlier than in several years following the catastrophe. Moreover, according to the recent data based on long-term study of the consequences of exposure to ionizing radiation in LSS, radiation-associated excess risks are recognized as persisting throughout the follow-up period up to 55 years after the exposure (and may be even longer) [3, 11]. Even after 45-55 years after exposure, the radiation-associated leukemia risk at 1 Gy was estimated to be twice the baseline risk [3]. This conclusion is of paramount importance for the follow-up of hematological malignancies in a cohort of Chernobyl clean-up workers.

In general, the incidence of radiation-associated leukemias increased with decreasing age at the time of A-bombing. LSS has also demonstrated that the risk associated with radiation exposure as well as shapes of the dose-response curves and the effects of age and gender varied significantly among the major types of leukemia proved as radiation-induced, namely CML, ALL and AML [6]. For ALL, risk appeared higher for childhood exposures and declined over time. For CML risk also declined over the time but the age at exposure was not found to have a significant effect on risk. The dose-response pattern for AML was less dependent on age at exposure or time since exposure as compared with ALL or CML. Moreover, contrary to ALL and CML, the excess rates for $A M L$ seem to rise with increasing attained age for those exposed as adults [3].

According to retrospective study, the explosion of A-bomb also led to an increased incidence of myelodysplastic syndromes (MDS), which occurred later than AML. The increasing rates of leukemias and MDS in Hiroshima and Nagasaki correlated with dose levels within 0.1-0.5 Gy based on the latest revision of dosimetry system $[12,13]$.

\section{FINDINGS, ESTIMATIONS \\ AND PREDICTIONS MADE BY VARIOUS RESEARCH TEAMS ON LEUKEMIA AND LYMPHOMA INCIDENCE IN CHERNOBYL CLEAN-UP WORKERS}

A cohort of Chernobyl clean-up workers of 19861987 provides another large massive of data accessible for the analysis of association between leukemias and the exposure to ionizing radiation. In total, more than 2.3 million people in Ukraine are included to the State Registry of Ukraine for people who suffered due to Chernobyl disaster (as of January 1, 2011); among them, more than 260 thousand clean-up workers are registered including more than 155 thousand people participating in emergency works in 1986. In Russia, 196 thousand clean-up workers are included into corresponding Registry.

Several research teams in Russia, Belarus and Ukraine as well as the multinational research teams have presented different estimates as to leukemia and lymphoma incidence in Chernobyl clean-up workers. Sometimes, however, the overall leukemia incidence in the exposed persons was taken into consideration without subdivision to the major biological types of the diseases. In most of the studies, only the major groups of leukemias such as AML, ALL (or even acute leukemias in total), and CML were delineated. Several recent studies have provided the evidence of the increased risk of leukemia and other hematological malignancies in Chernobyl clean-up workers. In fact, a number of authors have predicted and reported the increased incidence of leukemia among cleanup workers in Russia, Belarus and Ukraine [4, 10, 14], while the excess of leukemia was called in question elsewhere [7, 15].

A multinational case-control study of hematological malignancies among Chernobyl clean-up workers from Belarus, Russia and Baltic countries who had worked in Chernobyl in 1986-1987 provided by Kesminiene et al. [5] was one of the first such studies in which the individual dose reconstruction was attempted. RADRUE method of dose reconstruction developed for Chernobyl clean-up workers was used in this and subsequent studies [16]. 117 cases of malignancies of hematopoietic and lymphoid tissue (among them 69 leukemias, 34 cases of non-Hodgkin's Iymphoma (NHL), 8 cases of multiple myeloma (MM), 2 MDS and 4 cases of myeloproliferative disease, unclassifiable) were recorded in clean-up workers cohort with ERR per 10 cGy being of 0.60 . Such risk estimates were slightly higher than that calculated from data obtained in studies with A-bomb survivors and other low doserate studies. Among the malignancies in 70 cleanup workers whose dose was estimated with RADRUE, CLL incidence was $30.0 \%$ and $\mathrm{NHL}-28.6 \%$.

198 cases of leukemias were reported in the Russian cohort of clean-up workers; among them $24.3 \%$ all acute leukemias (without subdivision), 3.5\% MDS, $28.8 \%$ - CLL, 30.0\% - CML combined with chronic myelomonocytic leukemia and several other forms of leukemias [10]. According to Ivanov et al., the dynamics of standardized incidence ratio demonstrates a 2.5-3-fold increase in the leukemia incidence in the Russian cohort of Chernobyl clean-up workers in 5-7 years after the accident [14]. According to the forecast presented by Tsyb et al. for the Russian cohort of clean-up workers of 1986-1987 with the average dose of 16 cGy, about 900 cases of leukemias are expected life-time with $18 \%$ of them being attributed to radiation exposure [17].

The data of a few studies analyzing leukemias in Ukrainian cohort of clean-up workers have been available. Prisyazhniuk et al. stated the statistically significant increment in observed-to-predicted ratio of leukemia and lymphoma incidence: 2.6 in 19901993 and 2.0 in 1994-1997 [4]. In the report of Ukrainian-American nested case-control study of the cohort of 110,645 clean-up workers from the Chernobyl State Registry of Ukraine, 87 leukemia cases confirmed by the International panel of hematologists were presented [18]. Among them - 49 (48.9\%) cases of CLL, 4 - ALL, 6 - AML, 9 cases of acute leukemia other- 
wise not specified, $15-\mathrm{CML}$, and 4 cases of large granular lymphocytic leukemia (LGL). ERR of total leukemia was estimated as 3.44 per Gy.

Unfortunately, in most studies in post-soviet countries including Ukraine, the risks of increasing incidence of leukemias are based on the primary data categorized according to the obsolete ICD-9 (1975) classification where the acute leukemias have been registered in total without delineation of myeloid and lymphoid forms as well as cytological and immunophenotypic variants. Moreover, until recently only cytomorphology and several cytochemical techniques were routinely used for the diagnostic purposes in oncohematology in the vast majority of the hematological clinics in Ukraine.

\section{PATTERNS OF MALIGNANT DISEASES OF HEMATOPOIETIC AND LYMPHOID TISSUES IN UKRAINIAN CLEAN-UP WORKERS}

Precise diagnosis of the different types of hematological malignancies based on up-to-date classifications taking into account cytomorphological, immunophenotypical and molecular genetic features has contributed largely to our insight into radiationinduced leukemogenesis. The origin of one or another biological subtypes and cytological variants of leukemia seems to be specified by the effects of ionizing radiation on the pluripotent hematopoietic stem cells or the various categories of hematopoietic progenitor cells. Therefore, the comparative analysis of the patterns of oncohematological pathologies developed many years following the exposure of ionizing radiation in large cohorts of exposed persons is a topical issue of studies in Japanese A-bomb survivors, in Chernobyl clean-up workers and in residents of the areas contaminated with radionuclides as a consequence of Chernobyl disaster.

In 1993, the Reference Laboratory was set up as a public service at R.E. Kavetsky Institute of Experimental Pathology, Oncology and Radiobiology of the National Academy of Sciences of Ukraine with the aim of the precise diagnosis of the hematopoietic malignancies based on cytomorphology, cytochemistry, immunophenotyping in accordance with FAB, WHO, EGIL, ICD-10 and ICD-0-4 classifications. One of the principal tasks of the Reference Laboratory consisted in the systematic study of the malignant diseases of hematopoietic and lymphoid tissues in the major categories of people suffered due to Chernobyl catastrophe.

Since 1996, about 450 Ukrainian Chernobyl cleanup workers from Kyiv city and majority of the regional hospitals of Ukraine with suspected oncohematological disorders were referred to Reference Laboratory for confirmation and verification of the diagnosis. In 1996-2010, 295 cases of various types of leukemia were diagnosed and categorized in this group according to up-to-date classifications. In addition, in 118 patients among Chernobyl clean-up workers referred to Reference Laboratory non-malignant hematological diseases (aplastic anemia, hemolytic anemia, idiopathic thrombocytopenia, neutrophylic leukocytosis, lymphocytosis, dysgranulocytopoiesis, etc.) were ascertained. The year-by-year number of leukemia patients among Chernobyl clean-up workers first diagnosed in the Reference Laboratory is represented in Fig. 1. As shown in Fig. 2, more than half of the patients in this group were diagnosed at the age above 60 . All of them have been exposed to radiation in the course of emergency works at the age of 25-40. While the exact individual dosimetry data were lacking in most cases (as in most other Chernobyl studies), the estimated radiation dose load of the clean-up workers under study varied from 75 to $250 \mathrm{mGy}$. The main forms and cytological variants of hematological malignancies were diagnosed according to FAB-classification and REAL classification schemes. In 2001-2010, the diagnostic findings were revised in accordance with recently published new WHO classification of tumors of hematopoietic and lymphoid tissues [19].

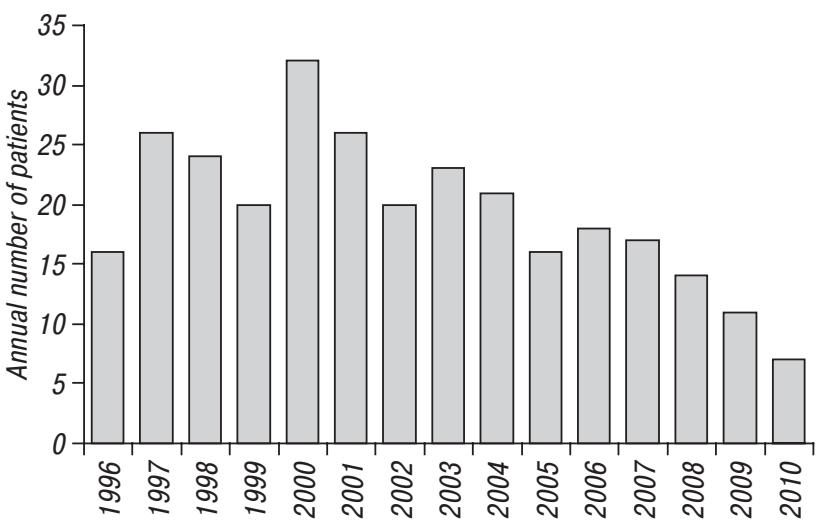

Fig. 1. Number of leukemia patients among clean-up workers first diagnosed in the Reference Laboratory in 1996-2010

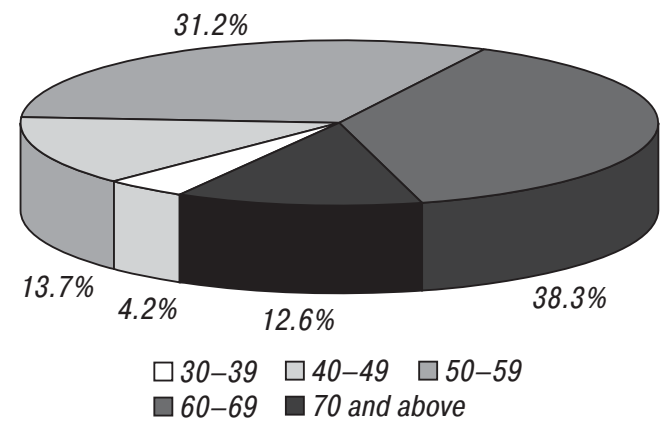

Fig. 2. Age distribution of leukemia patients among cleanup workers diagnosed in the Reference Laboratory in 1996-2010

The data on leukemia among Ukrainian Chernobyl clean-up workers diagnosed in 1996-2010 (1025 years after Chernobyl catastrophe) were compared with the data on 2697 consecutive patients referred to the laboratory for diagnosing from various regions of Ukraine within the same period without any selection of cases. The data on the phenotypes of leukemias in the group of clean-up workers and the patients who have not been explicitly exposed to ionizing radiation as well as the comparison of the percentage of the various biological types of leukemia in both groups are given elsewhere [20].

All the main types of tumors of hematopoietic and lymphoid tissues including B-CLL have been diag- 
nosed. The summary of all leukemia cases diagnosed among Chernobyl clean-up workers (295 cases) are given in Table together with the most recent data on leukemia patterns among LSS cohort ( 1215 cases in total) [3]. The general distribution of different biological subtypes of leukemia in Chernobyl cleanup workers and patients in LSS is quite similar.

Table. Absolute numbers and percentage of the major forms of tumors of hematopoietic and lymphoid tissues registered in cohort of LSS study up to 55 years after exposure [3] and in Chernobyl clean-up workers according to the files of Reference Laboratory in 10-25 years after exposure [20]

\begin{tabular}{lcc}
\hline \multicolumn{1}{c}{ Types of hematological malignancies } & LSS, $\mathrm{n}(\%)$ & $\begin{array}{c}\text { Clean-up } \\
\text { workers, } \mathrm{n}(\%)\end{array}$ \\
\hline AML & $229(18.85)$ & $46(15.60)$ \\
ALL & $62(5.10)$ & $17(5.76)$ \\
Other leukemias including acute leukemia & $26(2.14)$ & - \\
not otherwise specified & $99(8.15)$ & $27(9.15)$ \\
CML & - & $23(7.79)$ \\
Other myeloproliferative neoplasms & - & $10(3.39)$ \\
Chronic myelomonocytic leukemia & - & $16(5.42)$ \\
MDS & $16(1.32)$ & $77(26.10)$ \\
CLL & $501(41.23)$ & $35(11.86)$ \\
NHL & $42(3.46)$ & - \\
Hodgkin's lymphoma & $181(14.90)$ & $19(6.46)$ \\
MM & - & $15(5.08)$ \\
Other mature B-cell neoplasms & - & $10(3.39)$ \\
Mature T-cell neoplasms (including T-cell & - & - \\
LGL leukemia) & $59(4.85)$ & 295 \\
Adult T-cell leukemia & 1215 & \\
Total & &
\end{tabular}

When calculated cumulatively, the total percentage of three major forms of leukemias thought to be radiation-associated, namely ALL, AML, and CML, is similar in patients of LSS cohort and Chernobyl cleanup worker patients under the present study $(32.10 \%$ and $30.51 \%$, respectively) (see Table). Several discrepancies are quite obvious due to the different purposes and designs of these studies.

MDS has not been specially delineated in LSS [3], while this entity is believed to be associated with radiation in other studies based on LSS and ABDI cohorts [21]. MDS risk showed a significant linear response to exposure dose being also significantly greater in those exposed when young [22]. Moreover, according to Iwanaga et al., 42 of 151 MDS patients under follow-up study progressed to overt leukemia [21]. In our study, in the group of clean-up workers the morphological features of myelodysplasia were detected in 7 (15.2\%) of 46 patients with various cytological variants of AML, while in AML patients among non-exposed population of Kyiv city and Kyiv region such features of myelodysplasia were found only in $1.5 \%$ of all AML cases. This suggests that in the patients exposed to ionizing radiation the overt AML more frequently develops in the setting of preceding MDS [20]. Further studies are needed to clarify whether MDS is in fact associated with exposure to radiation taking into account gross underestimation of these pathologies in Ukraine. Much longer follow-up seems to be required for a correct estimate of radiationassociated risks of MDS and its conversion into overt acute leukemia.

Recent epidemiological data suggest the possible association between radiation exposure and CLL [5, 23]. These novel data contradict the previous ones including those in LSS $[3,6]$. Evaluation of CLL radiogenicity is hampered by a priori exclusion of CLL from many analyses reasoning that in other studies CLL has not been proved as radiation-associated leukemia [24]. It should be also taken into account that the classic LSS analyses are based on Japanese population wherein CLL is rather rare form of leukemia, while in USA and Europe B-CLL represents one of the most prevalent forms of leukemia in adults. The relatively low percentage of NHL in our study [20] is explained by the fact that only NHL cases in leukemization phase have been recorded in Reference Laboratory. In our previous study, we have demonstrated the tendency for increased $\mathrm{MM}$ rate among leukemias originating in Chernobyl clean-up workers [20]. The trend of the increased MM incidence was observed in epidemiological study in the contaminated areas of Belarus [25]. While earlier LSS data presented the evidence for increased MM risk among exposed persons [26], further LSS studies have not confirmed radiogenicity of MM [3]. Since MM, like CLL, is a malignant proliferative disorder of B-cell lineage, the novel data on CLL radiogenicity require the thorough consideration of $\mathrm{MM}$ rate among exposed persons in different populations.

The high incidence of LGL-leukemia among cleanup workers with hematopoietic malignancies $(1.69 \%$ vs $0,11 \%$ in non-exposed population [20]) is of particular importance since this category of T-cell and NK-cell neoplasms has not been revealed earlier in Ukraine. High incidence of neutropenia due to NK or NK-like $\mathrm{T}$-cell proliferative disorders was found among atomic bomb survivors in Japan [27].

To sum up, the studies in populations of Hiroshima and Nagasaki victims and Chernobyl clean-up workers revealed very broad spectrum of radiation-related hematological malignancies despite the differences among the types of exposure, dose rate, differences in age-sex structure of the exposed populations, etc. The following epidemiological surveys should be supplemented with studies based on molecular biology including the search for the specific radiogenic markers in leukemic cells. Moreover, the data on the tumors of hematopoietic and lymphoid tissues in the exposed persons after Chernobyl catastrophe may be very important for the prognostic estimates of the health effects of the Fukushima disaster.

\section{REFERENCES}

1. Effects of A-bomb radiation on the human body. I Shigenatsu, Ch Ito, N Kamada, M Akiyana, H Sasaki, eds. Tokyo: Harward Acad Publ, 1995. 419 p.

2. Ozasa K, Shimizu Y, Suyama A, et al. Studies of the mortality of atomic bomb survivors, report 14, 1950-2003: an overview of cancer and noncancer diseases. Radiat Res 2012; 177: 229-43.

3. Hsu WL, Preston, DL, Soda M, et al. The incidence of leukemia, lymphoma and multiple myeloma among atomic bomb survivors: 1950-2001. Radiat Res 2013; 179: 361-82.

4. Prisyazhniuk AE, Gryschenko VG, Fedorenko ZP, et al. Epidemiological study of cancer in population affected after the Chernobyl accident. Results, problems and perspectives. Int J Radiat Med 1999; 2: 42-50. 
5. Kesminiene A, Evrard AS, Ivanov VK, et al. Risk of hematological malignancies among Chernobyl liquidators. Radiat Res 2008; 170: 721-35.

6. Preston DL, Kusumi S, Tomonaga M, et al. Cancer incidence in atomic bomb survivors. Part III: Leukemia, lymphoma and multiple myeloma, 1950-1987. Radiat Res 1994; 137 (2 Suppl): S 68-97.

7. Report of the United Nations Scientific Committee on the Effects of Atomic Radiation to the General Assembly. UNSCEAR Report 2000. United Nations, New-York. 17 p.

8. Vrijheid $\mathrm{M}$, Cardis $\mathrm{E}$, Ashmore $\mathrm{P}$, et al. Ionizing radiation and risk of chronic lymphocytic leukemia in the 15-country study of nuclear industry workers. Radiat Res 2008; 170: 661-5.

9. Krestinina L, Preston DL, Davis FG, et al. Leukemia incidence among people exposed to chronic radiation from the contaminated Techa River, 1953-2005. Radiat Environ Biophys 2010; 49: 195-201.

10. Ivanov VK, Tsyb AF, Khait SE, et al. Leukemia incidence in the Russian cohort of Chernobyl emergency workers. Radiat Environ Biophys 2012; 51: 143-9.

11. Kodama K, Ozasa K, Okubo T. Radiation and cancer risk in atomic-bomb survivors. J Radiol Prot 2012; 32: 51-4.

12. Tomonaga $\mathrm{M}$, Nonaka $\mathrm{H}$, Matsuo $\mathrm{T}$. Atomic bomb irradiation and human leukemias. In: Nagasaki Symposium Radiation and Human Health. S Nagataki, S Yamashita, eds. Amsterdam: Elsevier, 1996: 197-215.

13. Finch SC. Myelodysplasia and radiation. Radiat Res 2004; 161: 603-6.

14. Ivanov VK, Tsyb AF, Gorsky AI, et al. Leukemia and thyroid cancer in emergency workers of Chernobyl accident: estimation of radiation risk (1986-1995). Radiat Environ Biophys 1997; 36: 9-16.

15. Rahu K, Hakulinen T, Smailyte $G$, et al. Site-specific cancer risk in the Baltic cohort of Chernobyl cleanup workers, 1986-2007. Eur J Cancer 2013; 49: 2926-33.

16. Kryuchkov V, Chumak V, Maceika E, et al. Radrue method for reconstruction of external photon doses for Chernobyl liquidators in epidemiological studies. Health Phys 2009; 97: 275-98.
17. Tsyb AF, Ivanov VK, Maksiutov MA. Consequences of Chernobyl catastrophe. In: Proc V Russian Oncol Conf Moscow, November 27-29, 2001 (http://www.rosoncoweb.ru/ library/congress/ru/05/09.php) (in Russian).

18. Romanenko AYe, Finch $S$, Hatch $M$, et al. The Ukrainian-American study of leukemia and related disorders among Chornobyl cleanup workers from Ukraine: III. Radiation risks. Radiat Res 2008; 170: 711-20.

19. WHO classification of tumours of haematopoietic and lymphoid tissues, $4^{\text {th }}$ ed. SH Swerdlow, E Campo, NL Harris, et al., eds. Lyon: IARC, 2008. 439 p.

20. Gluzman DF, Sklyarenko LM, Zavelevich MP, et al. Hematological malignancies in Chernobyl clean-up workers (1996-2010). J Hematol Malignancies 2012; 2: 43-50.

21. Iwanaga M, Hsu WL, Soda M, et al. Risk of myelodysplastic syndromes in people exposed to ionizing radiation: A retrospective cohort study of Nagasaki atomic bomb survivors. J Clin Oncol 2011; 29: 428-34.

22. Tsushima H, Iwanaga M, Miyazaki Y. Late effect of atomic bomb radiation on myeloid disorders: leukemia and myelodysplastic syndromes. Int J Hematol 2012; 95: 232-8.

23. Zablotska LB, Bazyka D, Lubin JH, et al. Radiation and the risk of chronic lymphocytic and other leukemias among Chornobyl cleanup workers. Environ Health Perspect 2013; 121: 59-65.

24. Silver SR, Hiratzka SL, Schubauer-Berigan MK, Daniels RD. Chronic lymphocytic leukemia radiogenicity: a systematic review. Cancer Causes Control 2007; 18: 1077-93.

25. National Report of Belarus. 20 years after Chernobyl catastrophe: Consequences in Republic of Belarus and actions for overcoming them. VE Chenchuk, VL Gurachevskii, eds. Commission on consequences of Chernobyl NPS catastrophe of Council of Ministers of Republic of Belarus, 2006. 112 p. (in Russian).

26. Shimizu Y, Kato H, Schull WJ. Life Span Study Report 11. Part 2. Cancer mortality in the years $1950-85$ based on the recently revised doses (DS86). Radiat Res 1990; 121: 120-41.

27. Imamura N, Kimura A. Neutropenia among survivors of atomic bomb explosion. Lancet 2000; 355: 117. 